A C G

Rec. Nat. Prod. 15:4 (2021) 281-292

records of natural

products

publications

\title{
Biotransformation of Perrottetin F by Aspergillus niger: New Bioactive Secondary Metabolites
}

\section{Danka Bukvicki $\oplus^{1,2,3^{*}}$, Miroslav Novakovic $\oplus^{2,4}$, Tatjana Ilic-Tomic $\oplus^{5}$, Jasmina Nikodinovic-Runic $\oplus^{5}$, Nina Todorovic $\oplus^{4}$, Milan Veljic $\oplus^{1}$ and Yoshinori Asakawa}

\author{
${ }^{1}$ University of Belgrade, Faculty of Biology, Institute of Botany and Botanical Garden \\ "Jevremovac", 11000 Belgrade, Serbia \\ ${ }^{2}$ Tokushima Bunri University, Faculty of Pharmaceutical Sciences Yamashiro-cho, Tokushima \\ 770-8514, Japan \\ ${ }^{3}$ University of Bologna, Department of Agricultural and Food Sciences, Via Fanin 46, 40127 \\ Bologna, Italy \\ ${ }^{4}$ University of Belgrade, Institute of Chemistry Technology and Metallurgy, Department of \\ Chemistry, 11000 Belgrade, Serbia \\ ${ }^{5}$ University of Belgrade, Institute of Molecular Genetics and Genetic Engineering, 11000 \\ Belgrade, Serbia
}

(Received October 14, 2020; Revised November 29, 2020; Accepted December 01, 2020)

\begin{abstract}
Biotransformation of bis-bibenzyl perrottetin F (1), isolated from the liverwort Lunularia cruciata by Aspergillus niger, has been investigated. New metabolites (2-4) have been isolated using reversed phase semipreparative HPLC and their structures were established to be 8-hydroxyperrottetin F, C-7-C-8 cleaved product, and perrottetin F 6'-sulfate using 1D and 2D NMR, HR-ESI-MS, IR and UV spectroscopy. The antimicrobial and cytotoxic properties of these compounds were also evaluated. Given the suggested cytotoxic properties of the parent compound, antiproliferative activity against healthy human lung fibroblasts (MRC5) and human lung carcinoma (A549) of three metabolites were evaluated revealing their lower cytotoxic properties in comparison to the starting compound - perrottetin F. The antimicrobial properties of these compounds were also evaluated, with the inhibitory activity against the Pseudomonas aeruginosa PAO1 and Staphylococcus aureus determined between $100 \mu \mathrm{M}$ and $450 \mu \mathrm{M}$. The metabolites showed remarkable ability to inhibit synthesis of bacterial quorum-sensing signal molecules such as short chain acyl homoserine lactones (AHLs). Therefore, biotransformation method represents fast and effective tool for obtaining new bioactive structures.
\end{abstract}

Keywords: Biotransformation; perrottetin F; liverworts; Aspergillus niger; cytotoxic activity; 1D and 2D NMR. (C) 2020 ACG Publications. All rights reserved.

\section{Introduction}

The Marchantiophyta (liverworts) produce various aromatic compounds among them bisbibenzyls, such marchantin, riccardin and perrottetin series which are very specific and rare natural products found only in this division. Up to date, 103 cyclic and acyclic bis-bibenzyls have been isolated from the liverworts [1-5]. Bis-bibenzyl skeleton comprises four aromatic rings (A-D),

\footnotetext{
* Corresponding author: E-Mail: dankabukvicki@gmail.com Phone:+381-63-16-11-980 Fax: 38-111-324-3603.
} 
biosynthesized from dimerization of lunularic acid trough dihydrocoumaric acid and prelunularin [5]. Perrottetin F (1) has been found in the thalloid liverworts Radula perrottetii, R. kojana and Lunularia cruciata $[4,6]$. This acyclic bis-bibenzyl was obtained as the major component from the methanol extract of L. cruciata [7]. Bis-bibenzyls possess wide range of biological activities such as cytotoxicity, 5- lipoxygenase and NO production inhibitory activity, antitumor, farnesoid X receptor (FXR)-activating activity, antimicrobial and many others [8,9]. Anti-influenza PA endonuclease potential of perrottetin $\mathrm{F}$ is described previously [10].

Known as 'green technology', biotransformation is used for converting various compounds into derivatives with possible biological activities [11]. Microbiological transformation of natural compounds can be useful for pharmaceutical drug development, in order to obtain more active compounds or to increase the yields of the pharmaceutical active components. It is an important strategy in the structural modification of natural compounds by using microorganisms, animal tissues or enzymes with great potential to produce novel, more efficient compounds [12]. Compounds obtained by biotransformation may improve properties in comparison to the starting component. It may also be good alternative to chemical synthesis of new drugs [13]. Literature data mainly show biotransformation of phytosterols, steroids, terpenes, alkaloids, and flavonoids [14]. It was noted that bacteria made complete steroid structure degradation while, micromycetes were active in multiple steroid hydroxylations. Some diterpenes (stemodin, stemodinone and stemarin) were biotransformed by A. niger to give three hydroxylated compounds [15]. Sesquiterpene cinnamodial was biotransformed to lactonized products [16].

In this study, an easy and cheap method for obtaining bioactive bis-bibenzyl derivatives using micromycete A. niger is presented. As per authors best knowledge there is only one report on biotransformation of bis-bibenzyl [9] and this is the first investigation on perrottetin $F$ (1) biotransformation, from which three new derivatives were obtained, and their biological activities were investigated.

\section{Materials and Methods}

\subsection{General Experimental Procedures}

Optical rotations were measured on a Rudolph Research Analytical Autopol IV automatic polarimeter with methanol as solvent and concentration of compounds $1.00 \mathrm{mg} / \mathrm{mL}$. UV spectra were recorded on a GBC Cintra UV/VIS spectrometer with methanol as solvent in concentration range 3-9 $\times 10^{-5} \mathrm{M}$. IR spectra were obtained on a Thermoscientific Nicolet 6700 FT-IR spectrometer. NMR spectra were measured on a Bruker Avance III 500 spectrometer at $500.26 \mathrm{MHz}$ for ${ }^{1} \mathrm{H}$ and 125.80 $\mathrm{MHz}$ for ${ }^{13} \mathrm{C}$, with $\mathrm{CD}_{3} \mathrm{OD}$ as a solvent. HR-ESI-MS data were obtained on Agilent 6210 Time-ofFlight LC/MS system equipped with an ESI interface (ESITOFMS). Solvent was methanol, mobile phase was: $0.2 \% \mathrm{HCOOH} / \mathrm{CH}_{3} \mathrm{CN} 1: 1,0.2 \mathrm{~mL} / \mathrm{min}$. The ESI was operated in a negative mode and nitrogen was used as the drying gas $(12 \mathrm{~L} / \mathrm{min})$ and nebulizing gas at $350{ }^{\circ} \mathrm{C}(45 \mathrm{psi})$. The OCT RF voltage was set to $250 \mathrm{~V}$ and the capillary voltage was set to $4.0 \mathrm{kV}$. The voltages applied to the fragmentor and skimmer were $140 \mathrm{~V}$ and $60 \mathrm{~V}$, respectively. Scanning was performed from 100 to $1500 \mathrm{~m} / \mathrm{z}$ (mass-to-charge ratio). For column chromatography (CC) silica gel $60\left(\mathrm{SiO}_{2} ; 0.063-0.200\right.$ $\mathrm{mm}$, Merck) was used. Analytical TLC was carried out on silica gel 60 GF254 $20 \times 20 \mathrm{~cm}$ plates, with a layer thickness of $0.25 \mathrm{~mm}$ (Merck). Preparative TLC was carried out on silica gel $60 \mathrm{GF} 254\left(\mathrm{SiO}_{2}\right.$; $<0.063 \mathrm{~mm}$, Merck) packed $20 \times 20 \mathrm{~cm}$ plates, thickness $0.75 \mathrm{~mm}$. Semipreparative HPLC separation was performed on an Agilent Instrument 1100 series equipped with a DAD (Agilent Technologies, G1315C). The column used was a Zorbax Eclipse XDB C18 $(9.4 \mathrm{~mm} \times 250 \mathrm{~mm}, 5 \mu \mathrm{m})$. Solvents for HPLC separation were of chromatographic grade. Solvents for CC were freshly distilled.

\subsection{Plant material, Extraction and Isolation}

A voucher specimen (No. 17290) has been deposited in the Herbarium at the Institute of Botany and Botanical Garden "Jevremovac", University of Belgrade (BEOU). Extraction and 
fractionization of crude methanol extract of liverwort Lunularia cruciata (L.) Dumort. ex Lindb. (Lunulariaceae) by silica gel CC and isolation of dominant compound perrottetin F have been done according to previously described procedure [7].

\subsection{Aspergillus niger TBUYN-2 Growth and Biotransformation Procedure}

Micromycete Aspergillus niger TBUYN-2 obtained from the soil collected in Osaka prefecture [9] was grown in Czapek-peptone medium $(\mathrm{pH} 7)$ in incubator shaker at $120 \mathrm{rpm}, 25^{\circ} \mathrm{C}$ for 3 days. When mycromicete achieved full growth, perrottetin $\mathrm{F}(\mathbf{1})(50 \mathrm{mg}$ was dissolved in $0.5 \mathrm{~mL}$ of ethanol) was added into three flasks (150 mg in total) and left in thermoshaker at $120 \mathrm{rpm}, 25^{\circ} \mathrm{C}$ for 4 days. EtOH $(0.5 \mathrm{~mL})$ was added in the flask with A. niger and medium, as control.

After 4 days, medium and mycelia were separated in a Buchner separation funnel. For extraction of compounds from media EtOAc was used, while mycelia were left in acetone for next 7 days. $\mathrm{pH}$ of media was 3.5. To get basic-neutral fraction (BNF) from media and mycelia (pH 9) 50\% $\mathrm{Na}_{2} \mathrm{CO}_{3}$ was added. Acidic fraction (AF) ( $\mathrm{pH} 2-3$ ) was obtained by adding $5 \% \mathrm{H}_{2} \mathrm{SO}_{4}$ solution in media. After extraction analytical TLC was carried out on aluminium silica sheet gel $60 \mathrm{~F}_{254}$ (MERCK). Chromatograms were developed using $\mathrm{CH}_{2} \mathrm{Cl}_{2}$ : EtOH $(7: 1 \mathrm{v} / \mathrm{v})$ as the developing solvent. The presence of biotransformed compounds were detected under UV light $(254 \mathrm{~nm})$ and spraying the $30 \% \mathrm{H}_{2} \mathrm{SO}_{4}$ solution on TLC sheet. The biotransformation products were separated using semipreparative HPLC.

\subsection{Cytotoxicity Assay}

Cytotoxicity of compounds (1-4) was measured using the methods described previously [17]. A549 (human lung carcinoma, obtained from ATCC) and MRC5 cells (human lung fibroblast, obtained from ATCC) were plated in a 96-well flat-bottom plate at a concentration of $1 \times 10^{4}$ cells per well, grown in humidified atmosphere of $95 \%$ air and $5 \% \mathrm{CO}_{2}$ at $37{ }^{\circ} \mathrm{C}$, and maintained as monolayer cultures in RPMI-1640 medium supplemented with $100 \mu \mathrm{g} / \mathrm{mL}$ streptomycin, $100 \mathrm{U} / \mathrm{mL}$ penicillin, and $10 \%(\mathrm{v} / \mathrm{v})$ fetal bovine serum (FBS). After $24 \mathrm{~h}$ of A549 and MRC5 cells incubation, the media containing increasing concentrations of each tested compound (with the highest concentration tested being $500 \mu \mathrm{M}$ ) were added to the cells. Stock solutions of bis-bibenzyls were prepared in DMSO (50 $\mathrm{mM})$. Control cultures received the solvent DMSO and blank wells contained $200 \mu \mathrm{L}$ of growth medium. After $48 \mathrm{~h}$ of incubation, cells proliferation was determined using 3-(4,5-dimethylthiazol-2yl)-2,5-diphenyltetrazolium bromide (MTT) reduction assay. MTT solution $(0.5 \mathrm{mg} / \mathrm{mL})$ was added after treatment and incubated for $1 \mathrm{~h}$, followed by dimethyl sulfoxide incubation. Cell proliferation was determined from the absorbance (A) at $540 \mathrm{~nm}$ on Tecan Infinite 200 Pro multiplate reader (Tecan Group, Männedorf, Switzerland). The MTT assay was performed two times in quadruplicate and the results were presented as percentage of the control (untreated cells) that was arbitrarily set to $100 \%$. The cell viability rate (\%) was calculated as follows: (A of the treated group/A control group) $\times 100$.

\subsection{Antibacterial Activity Assays}

Pseudomonas aeruginosa PAO1 ATCC 27853, Staphylococcus aureus ATCC 25923 and S. marcescens ATCC 27117 used in this study were from the American Type Culture Collection (ATCC). P. aeruginosa PAOJP2, P. aeruginosa PA14-R3, and PAO1DpqsAmini-CTXlux-PpqsA were kindly provided by Dr. Livia Leoni, Department of Biology, Roma Tre University, Italy. Chromobacterium violaceum CV026 [18] was provided by Prof. Vittorio Venturi (ICGB, Trieste, Italy). Bacteria were routinely grown in Luria-Bertani (LB) medium $(1 \%(\mathrm{w} / \mathrm{v}) \mathrm{NaCl}, 1 \%(\mathrm{w} / \mathrm{v})$ Tryptone, $0.5 \%(\mathrm{w} / \mathrm{v})$ yeast extract) with or without agar $(1.5 \% \mathrm{w} / \mathrm{v})$ with shaking $(180 \mathrm{rpm})$ at $37{ }^{\circ} \mathrm{C}$. Tryptone soy broth (TSB) (Oxoid) was used for growth of $P$. aeruginosa PAO1 in the assay of biofilm formation. Antibiotics kanamycin (Kanamycin sulfate from Streptomyces kanamyceticus, BioReagent, Sigma-Aldrich, purity $>95 \%$ ), streptomycin (Streptomycin sulfate salt, BioReagent, Sigma-Aldrich, purity >96\%) and tetracycline (BioReagent, Sigma-Aldrich, purity >96\%) were incorporated into growth medium at the concentration of $30 \mu \mathrm{g} / \mathrm{mL}$ or $200 \mu \mathrm{g} / \mathrm{mL}$ in the case of kanamycin, $20 \mu \mathrm{g} / \mathrm{mL}$ for streptomycin and $100 \mu \mathrm{g} / \mathrm{mL}$ for tetracycline. 


\subsubsection{Minimum Inhibitory Concentration (MIC) Determination}

The minimum inhibitory concentrations of bis-bibenzyls were determined according to standard broth microdilution assays recommended by the Clinical and Laboratory Standards Institute (M07A9). Stock solutions of bis-bibenzyls were prepared in DMSO $(50 \mathrm{mM})$. The highest tested concentration of each compound was $500 \mu \mathrm{M}$. The inoculums were $10^{5}$ colony forming units $(\mathrm{CFU}) / \mathrm{mL}$. The MIC value corresponds to the lowest concentration that inhibited the growth after $24 \mathrm{~h}$ at $37^{\circ} \mathrm{C}$.

\subsubsection{Inhibition of P. aeruginosa PAO1 Biofilm Formation}

P. aeruginosa biofilms were grown in TSB medium without shaking conditions. The effect of perrottetin F (1) and biotransformants on biofilm forming ability was tested at concentrations of $50 \%$ of determined MIC (0.5MIC) for each compound, on polystyrene flat-bottomed microtitre 96 well plates as previously described with modifications [19]. Briefly, overnight culture of $P$. aeruginosa was diluted in a fresh LB medium to optical density of OD600 nm=0.2 and $100 \mu \mathrm{L}$ of the diluted culture with appropriate compound at the concentration of $0.5 \mathrm{MIC}$ was added to the plate. After incubation for $24 \mathrm{~h}$ at $37^{\circ} \mathrm{C}$, the plate was washed twice with sterile water, dried, stained for 30 min with $0.1 \%$ crystal violet in order to determine biofilm formed. Stained cells were washed with sterile water to remove unbound crystal violet. After drying, $150 \mu \mathrm{L}$ of $30 \%(\mathrm{v} / \mathrm{v})$ acetic acid was added to solubilize the dye. After $10 \mathrm{~min}$, the content of the wells was homogenized, and the OD of samples were measured at $550 \mathrm{~nm}$ using microplate reader Infinite 200PRO (Tecan). The experiment was done in quintuplicate and repeated two times.

\subsubsection{Activity on Bacterial Pigment Production}

Effect of bis-bibenzyls on violacein synthesis was evaluated with Chromobacterium violaceum CV026 indicator strain, as reported previously $[18,20]$. Briefly, semisolid LB agar $(0.3 \%$, w/v; $5 \mathrm{~mL}$ ) was seeded with $50 \mu \mathrm{L}$ of an overnight culture of $C$. violaceum CV026 supplemented by $N$ hexanoyl- L-homoserine lactone (Sigma) to a final concentration of $5 \mu \mathrm{M}$ and poured over the surface of LB agar plates. When the overlaid agar had solidified, sterilized discs containing $250 \mu \mathrm{g}$ of each compound were placed on the plates. Petri dishes were incubated in the upright position overnight (30 ${ }^{\circ} \mathrm{C}$ ) and examined for violacein synthesis. Inhibition of violacein synthesis was defined by the presence of white haloes in a purple background.

Overnight culture of $S$. marcescens was diluted 100-fold in molten semi-solid LB agar (0.3\% w/v) and poured over solid LB medium. Cellulose discs containing compounds ( $250 \mu \mathrm{g} / \mathrm{disc})$ were placed on solidified agar and incubated for $24 \mathrm{~h}$ at $30{ }^{\circ} \mathrm{C}$. Inhibition of prodigiosin synthesis was identified by the absence of red colour around the disc.

\subsubsection{Activity on Acyl Homoserine Lactones (AHLs) and Alkyl Quinolones (AHQs) Production using Biosensors}

For the detection and quantification of long-chain (3OC12-HSL) and short-chain AHLs (C4HSL) in AHLs production assays with two $P$. aeruginosa biosensors based on luminescent bacteria: $P$. aeruginosa PA14-R3 $\Delta$ lasIPrsaI::lux and $P$. aeruginosa PAO1 $\Delta$ rhlIpKD-rhlA sensors for monitoring C12-AHL and C4-AHL are used, respectively [21, 22].

$P$. aeruginosa PA14-R3 $\Delta$ lasIPrsaI:lux and $P$. aeruginosa PAO1 $\Delta$ rhlIpKD-rhlA biosensors reporter strains were grown overnight at $37{ }^{\circ} \mathrm{C}$ on LA agar plate. Plate for P. aeruginosa PAO1 $\Delta$ rhlIpKD-rhlA was supplemented with kanamycin (Sigma), $200 \mu \mathrm{g} / \mathrm{mL}$. Bacterial colonies were resuspended in LB medium with or without appropriate antibiotic and overnight cultures of the biosensors were diluted to $\mathrm{OD} 600=0.045$ in LB. Bacterial suspension consists of $100 \mu \mathrm{L}$ of $P$. aeruginosa PA14-R3 $\Delta$ lasIPrsaI::lux sensor supplemented by 3OC12-HSL lactone (Sigma) to a final concentration of $5 \mu \mathrm{M}$ or the suspension consists of $100 \mu \mathrm{L}$ of $P$. aeruginosa PAO1 $\Delta$ rhlIpKD-rhlA sensor supplemented by C4-HSL (Sigma), to a final concentration of $5 \mu \mathrm{M}$, were dispensed in black, 
clear-bottom 96-well microtiter plates. Microtiter plates were incubated at $37{ }^{\circ} \mathrm{C}$ with shaking, after 4 $\mathrm{h}$ of incubation OD600 and bioluminescence were simultaneously measured on Tecan Infinite 200 Pro multiplate reader (Tecan Group Ltd., Männedorf, Switzerland). Luminescence values was normalized by the cell density and expressed as percentage of the corresponding untreated control values. All experiments were performed in triplicate at least three times.

For detection and quantification of quorum sensing molecules 2-alkyl-4-quinolones (AHQs), such as 2-heptyl -3-hydroxy-4 quinolone (PQS) and 2-heptyl-4-quinolone (HHQ), a lux-based $P$. aeruginosa AHQ sensor was employed in liquid microtiter plate assay. $100 \mu \mathrm{l}$ of $P$. aeruginosa PAO1 $\Delta$ pqsAmini-CTXluxPpqsA biosensor supplemented by HHQ lactone (Sigma), to a final concentration of $5 \mu \mathrm{M}$, was used for detection and quantification of AHQs molecules as described previously [23].

Compound 2: brown, amorphous solid; $[\alpha]_{\mathrm{D}}^{22}+16.0\left(c\right.$ 1.0, MeOH); UV $(\mathrm{MeOH}) \lambda_{\max }(\log \varepsilon) 212$ (4.54), 275 (3.76); IR (KBr) $v_{\max } 3413,2923$, 2855, 1600, 1506, 1456, 1352, 1342, 1322, 1257, 1215, $1168,1033,964,853,785 \mathrm{~cm}^{-1}$; ${ }^{1} \mathrm{H}$ NMR and ${ }^{13} \mathrm{C}$ NMR, see Table 1; HR-ESI-MS $\mathrm{m} / z$ 457.1668 [M $\mathrm{H}]^{-}$(calcd for $\mathrm{C}_{28} \mathrm{H}_{25} \mathrm{O}_{6}-\mathrm{H}, 457.1651$ ).

Compound 3: brown, amorphous solid; UV (MeOH) $\lambda_{\max }(\log \varepsilon) 224$ (3.56), 275 sh (4.32); IR (KBr) $v_{\max } 3452,2924,2854,1632,1507,1454,1384,1213,1166,1123,1083,1034,792,686 \mathrm{~cm}^{-1} ;{ }^{1} \mathrm{H}$ NMR and ${ }^{13} \mathrm{C}$ NMR, see Table 1; HR-ESI-MS $m / z 351.1244[\mathrm{M}-\mathrm{H}]^{-}$(calcd for $\mathrm{C}_{21} \mathrm{H}_{20} \mathrm{O}_{5}-\mathrm{H}$, $351.1233)$.

Compound 4: white, amorphous solid; UV (MeOH) $\lambda_{\max }(\log \varepsilon) 215$ (4.85), 272 (4.13), 282 (4.11); IR $(\mathrm{KBr}) v_{\max } 3413,2925,2857,1595,1590,1505,1455,1281,1242,1215,1166,1060,1021,999,856$, 780, 729, $695 \mathrm{~cm}^{-1}$; ${ }^{1} \mathrm{H}$ NMR and ${ }^{13} \mathrm{C}$ NMR, see Table 1; HR-ESI-MS $m / z 521.1283[\mathrm{M}-\mathrm{H}]^{-}$(calcd for $\mathrm{C}_{28} \mathrm{H}_{26} \mathrm{O}_{8} \mathrm{~S}-\mathrm{H}, 521.1270$ ).

\section{Results and Discussion}

\subsection{Isolation and Identification of A. niger Biotransformed Products of Perrottetin F}

From the EtOAc extract of basic-neutral fraction of the A. niger medium, after separation on reversed-phased semipreparative HPLC compounds $\mathbf{2}$ and $\mathbf{3}$ have been isolated. From the acetone extract of the mycelium, compounds $\mathbf{2}$ and $\mathbf{4}$ have been isolated.

Compound 2 was obtained as a brown amorphous substance. Molecular ion $[\mathrm{M}-\mathrm{H}]^{-}$at $\mathrm{m} / \mathrm{z}$ 457.1668 of its HR-ESI-MS spectrum and the ${ }^{13} \mathrm{C}$ NMR spectrum (Table 1, Figures S7, S10 and S11, Supporting Information) were in accordance with molecular formula $\mathrm{C}_{28} \mathrm{H}_{26} \mathrm{O}_{6}$. The UV absorption bands at 212 and $275 \mathrm{~nm}$ and the IR bands at 3413 and $1600 \mathrm{~cm}^{-1}$ indicated the presence of phenol moieties in 2. In the ${ }^{1} \mathrm{H}$ NMR spectrum signals of the A-, C-, and D-rings were very similar to those of perrottetin F (1) (Figures S1 and S8, Supporting Information). However, the signals of the B-ring protons (connected by the COSY spectrum, Figure S12, Supplementary Material) were downfield shifted. One proton signal appeared at $\delta_{\mathrm{H}}, 4.60(\mathrm{t}, 7.0 \mathrm{~Hz})$ and there were six "bridge" protons $\left(\delta_{\mathrm{H}} 2.5-\right.$ 2.9 region, Table 1) when compared with 1 . These data and the HSQC spectrum (Figure S15, Supporting Information), indicated the presence of a hydroxyl group at one of the four benzyl positions. Strong NOE correlations of the proton at $\delta_{\mathrm{H}} 4.60$ with $\mathrm{H}-10$ and $\mathrm{H}-14$, as well as weaker NOE correlations with H-3 and H-5 (Figure S14, Supporting Information) revealed that $\mathrm{OH}$ was at C8. The COSY correlations H-8/H-7a, H-7b and the HMBC correlations H-10, H-14/C-8 gave additional confirmation (Figure S13 and S18, Supporting Information). Thus, the structure of the first new bis-bibenzyl 2 was established as 8-hydroxyperrottetin F. Its absolute configuration remained to be clarified.

Compound 3 was isolated as a brown amorphous substance. Its molecular $[\mathrm{M}-\mathrm{H}]^{-}$ion at $\mathrm{m} / \mathrm{z}$ 351.1244 from the HR-ESI-MS and ${ }^{13} \mathrm{C}$ NMR spectrum (Figures S19 and S22, Supporting Information) revealed the molecular formula $\mathrm{C}_{21} \mathrm{H}_{20} \mathrm{O}_{5}$, i.e. seven $\mathrm{C}$ atoms less compared with compound 1. The UV absorption bands at 224 and $275 \mathrm{~nm}$ and the IR bands at 3452 and $1632 \mathrm{~cm}^{-1}$ indicated the presence of phenol moieties in 3. The 1D and 2D NMR data revealed the presence of 
three benzene rings with the hydroxyl group at one benzyl position. The singlet at $\delta_{\mathrm{H}} 4.45\left(\delta_{\mathrm{C}} 65.0\right)$ originated from the methylene group, suggested terminal hydroxyl substituent. The HMBC correlations of this singlet with the overlapped signals of C-3 and C-5 and NOE correlations $\mathrm{H}-3, \mathrm{H}-$ 5/H-7 (Figures S27 and S24, Supporting Information) referred to the 7-hydroxyl derivative. The rest of ${ }^{1} \mathrm{H}$ and ${ }^{13} \mathrm{C}$ NMR signals, very similar to those of the compounds $\mathbf{1}$ and $\mathbf{2}$, were ascribed to the $\mathrm{C}$ and $\mathrm{D}$ rings atoms (Figures S1, S8, S23-S25, Supporting Information). Thus, the structure of compound $\mathbf{3}$ was established as shown in Figure 1.<smiles>Oc1cccc(CCc2ccc(Oc3cccc(CCc4cc(O)c(O)c(Oc5cccc(CCc6cccc(O)c6)c5)c4)c3)cc2)c1</smiles>

Figure 1. Biotransformed products of perrottetin F (1) obtained by A. niger. 
Table 1. ${ }^{1} \mathrm{H}$ and ${ }^{13} \mathrm{C}$ NMR Data of Compounds 2-4 recorded in $\mathrm{CD}_{3} \mathrm{OD}\left(500 \mathrm{MHz}\right.$ for ${ }^{1} \mathrm{H}$ and $125 \mathrm{MHz}$ for $\left.{ }^{13} \mathrm{C}\right)$

\begin{tabular}{|c|c|c|c|c|c|c|}
\hline \multirow[b]{2}{*}{ Position } & \multicolumn{2}{|r|}{2} & \multicolumn{2}{|r|}{3} & \multicolumn{2}{|r|}{4} \\
\hline & $\delta \mathrm{C}$ & $\delta \mathbf{~}$ & $\delta \mathrm{C}$ & $\delta \mathbf{\delta H}$ & $\delta \mathrm{C}$ & $\delta \mathbf{~}$ \\
\hline 1 & 157.8 & - & 158.9 & - & 157.5 & - \\
\hline 2 & 118.2 & $6.61 \mathrm{~d}(8.5)$ & 118.3 & $6.74 \mathrm{~d}(8.5)$ & 118.3 & $6.75 \mathrm{~d}(8.5)$ \\
\hline 3 & 131.7 & $6.90 \mathrm{~d}(8.5)$ & 129.7 & $7.17 \mathrm{~d}(8.5)$ & 130.7 & $7.07 \mathrm{~d}(8.5)$ \\
\hline 4 & 134.0 & - & 136.7 & - & 137.3 & - \\
\hline 5 & 131.7 & $6.90 \mathrm{~d}(8.5)$ & 129.7 & $7.17 \mathrm{~d}(8.5)$ & 130.7 & $7.07 \mathrm{~d}(8.5)$ \\
\hline 6 & 118.2 & $6.61 \mathrm{~d}(8.5)$ & 118.3 & $6.74 \mathrm{~d}(8.5)$ & 118.3 & $6.75 \mathrm{~d}(8.5)$ \\
\hline 7 & 46.0 & $2.80 \mathrm{~m}$ & 65.0 & $4.45 \mathrm{~s}$ & 38.3 & $2.83 \mathrm{~m}$ \\
\hline 8 & 76.7 & $4.60 \mathrm{t}(7.0)$ & - & - & 39.3 & $2.80 \mathrm{~m}$ \\
\hline 9 & 147.5 & - & - & - & 144.8 & - \\
\hline 10 & 114.2 & $6.63 \mathrm{t}(2.0)$ & - & - & 116.5 & $6.62 \mathrm{t}(2.0)$ \\
\hline 11 & 158.4 & - & - & - & 158.5 & - \\
\hline 12 & 115.2 & $6.52 \mathrm{dd}(7.5 ; 2.0)$ & - & - & 113.9 & $6.59 \mathrm{~m}$ \\
\hline 13 & 130.3 & $6.96 \mathrm{t}(7.5)$ & - & - & 130.4 & $7.05 \mathrm{t}(8.0)$ \\
\hline 14 & 118.7 & $6.61 \mathrm{~m}^{\mathrm{b}}$ & - & - & 121.0 & $6.64 \mathrm{~m}$ \\
\hline $1^{\prime}$ & 136.1 & - & 136.4 & - & 140.9 & - \\
\hline $2^{\prime}$ & 145.6 & - & 145.3 & - & 142.6 & - \\
\hline $3^{\prime}$ & 112.7 & $5.97 \mathrm{~d}(2.0)$ & 113.0 & $6.03 \mathrm{~d}(2.0)$ & 118.9 & $6.43 \mathrm{~d}(2.0)$ \\
\hline $4^{\prime}$ & 134.3 & - & 134.4 & - & 134.1 & - \\
\hline $5^{\prime}$ & 112.4 & $6.33 \mathrm{~d}(2.0)$ & 112.8 & $6.38 \mathrm{~d}(2.0)$ & 119.5 & $7.05 \mathrm{~d}(2.0)$ \\
\hline $6^{\prime}$ & 147.8 & - & 148.0 & - & 146.2 & - \\
\hline $7^{\prime}$ & 38.4 & $2.56 \mathrm{~m}$ & 38.5 & $2.60 \mathrm{~m}$ & 38.3 & $2.75 \mathrm{~m}$ \\
\hline $8^{\prime}$ & 39.1 & $2.56 \mathrm{~m}$ & 39.2 & $2.60 \mathrm{~m}$ & 39.0 & $2.75 \mathrm{~m}$ \\
\hline $9^{\prime}$ & 144.6 & - & 144.6 & - & 144.4 & - \\
\hline $10^{\prime}$ & 116.5 & $6.40 \mathrm{t}(2.0)$ & 116.6 & $6.47 \mathrm{dd}(8.0 ; 2.0)^{\mathrm{c}}$ & 116.6 & $6.55 \mathrm{t}(2.0)$ \\
\hline $11^{\prime}$ & 158.3 & - & 158.4 & - & 158.4 & - \\
\hline $12^{\prime}$ & 113.8 & $6.43 \mathrm{~d}(8.0)$ & 113.9 & $6.43 \mathrm{t}(2.0)$ & 113.9 & $6.57 \mathrm{dd}(7.5 ; 1.0)$ \\
\hline$\underline{13^{\prime}}$ & $\underline{130.2}$ & $6.89 \mathrm{t}(8.0)$ & 130.3 & $6.92 \mathrm{t}(7.5)$ & 130.4 & $\underline{7.02 \mathrm{t}(8.0)}$ \\
\hline $14^{\prime}$ & 121.0 & $6.43 \mathrm{dd}(8.0 ; 2.0)$ & 121.0 & $6.47 \mathrm{dd}(8.0 ; 2.0)^{\mathrm{c}}$ & 121.0 & $6.60 \mathrm{~m}$ \\
\hline
\end{tabular}

${ }^{a} J$ values are given in parenthesis

${ }^{b}$ signals overlayed with signals for $\mathrm{H}-2$ and H-6

${ }^{c}$ signals overlayed

For compound 4, obtained as a white amorphous substance, two molecular formulas $\mathrm{C}_{28} \mathrm{H}_{26} \mathrm{O}_{8} \mathrm{~S}$ and $\mathrm{C}_{28} \mathrm{H}_{26} \mathrm{O}_{10}$ were possible regarding the molecular ion $[\mathrm{M}-\mathrm{H}]^{-}$at $\mathrm{m} / \mathrm{z} 521.1283$ from the HR-ESI-MS spectrum and the ${ }^{13} \mathrm{C}$ NMR spectrum (Figures S28 and S30, Supporting Information). Calculating isotopic distribution and percentage of the $[\mathrm{M}+2-\mathrm{H}]^{-}$ion from the HR-ESI-MS spectrum, our experimental value of $9.1 \%$ was close to the theoretical value of $10.9 \%$ for the $[\mathrm{M}+2-\mathrm{H}]^{-}$ion for the molecular formula $\mathrm{C}_{28} \mathrm{H}_{26} \mathrm{O}_{8} \mathrm{~S}$. In the case of $\mathrm{C}_{28} \mathrm{H}_{26} \mathrm{O}_{10}$, [M+2-H] ion prediction was $6.7 \%$. NMR analyses revealed great similarity of ${ }^{1} \mathrm{H}$ NMR spectrum of $\mathbf{4}$ to those of its biotransformation parent compound perrottetin $\mathrm{F}(\mathbf{1})$. The ${ }^{1} \mathrm{H}$ NMR spectrum of $\mathbf{4}$ was almost identical to that of perrottetin $\mathrm{F}$ (1) (Figures S29 and S1, Supporting Information), except the H-3' and H-5' signals of 4 that were downfield shifted (for 0.3 and $0.6 \mathrm{ppm}$, respectively), in comparison to compound $\mathbf{1}$. This indicated another substituent at C-6' position, since multiplicity of both, H-3' and H-5', remained the same (two doublets with $J=2.0 \mathrm{~Hz}$ ). The difference of chemical shifts of C-ring carbons between 4 and perrottetin F (especially C-3', C-5', but also C-1', C-2' and C-6') additionally confirmed significant change in Cring (Figures S30 and S3, Supporting Information). Having in mind the presence of sulphur in 4, ie. the molecular formula $\mathrm{C}_{28} \mathrm{H}_{26} \mathrm{O}_{8} \mathrm{~S}$, it was concluded that sulfate group was at $\mathrm{C}-6$ ' position instead of $\mathrm{OH}$ group present in perrottetin F (1). All 2D NMR correlations confirmed the structure of perrottetin F-6'-sulfate (4) (Figures S31-S36, Supporting Information).

Although this is the first time that biotransformation of perrottetin $F(\mathbf{1})$ was investigated, the obtained compounds are in agreement with literature data for the similar compounds obtained by the 
microbial biotrasformation using A. niger. The "target" positions in molecules during A. niger action were mostly allylic or benzyl C-atoms, or double bonds [24]. Successful biotransformation of thymol at the benzyl position by A. niger was reported [25]. In this work, compound $\mathbf{2}$ was obtained by hydroxylation at benzyl position $\mathrm{C}-8$, and $\mathbf{3}$ by oxidative cleavage at the benzyl position C-7. Additionally, sulfate derivatives after Aspergillus spp. biotransformation were previously reported [26] and are in accordance with our findings (compound $\mathbf{4}$ ).

\subsection{Biological Activity Evaluations of Three Biotransformation Products in Comparison to Perrottetin $F(1)$}

The cytotoxic activity (antiproliferative effect) of biotransformants was evaluated against two cell lines and compared to perrottetin F (1) (Table 2). Compounds (2-4) obtained by biotransformation of perrottetin $\mathrm{F}$ were less effective in inhibiting the growth of human lung carcinoma (A549) as well as human healthy lung fibroblasts (MRC5) in comparison to the parent compound. The reduction was between 1.6- to 16.6-fold for A549 and 2.3- to 8.3-fold for MRC5 cells, with the 4 being the least and 2 being the most active amongst biotransformants (Table 2). Some literature data proved that present of sulfated groups may reduce cytotoxic potential of molecule [27]. For example, flavonoids luteolin and hispidulin showed higher cytotoxicity on melanoma cells line (B16F10) compared with luteolin-7sulphate and hispidulin 7-sulphate [27]. This can be reason of slightly lower activity of compound $\mathbf{4}$ in regard to perrottetin $\mathrm{F}$.

Biotransformed product 2 also showed the slight increase in the selectivity toward cancer in comparison to healthy cell line compared to perrottetin F.

Table 2. The cytotoxicity $\left(\mathrm{IC}_{50}, \mu \mathrm{M}\right)$ and antibacterial properties (MIC, $\mu \mathrm{M}$ ) of three biotransformation products in comparison to perrottetin $\mathrm{F}$

\begin{tabular}{ccccc}
\hline & \multicolumn{2}{c}{ IC $_{\mathbf{5 0}}(\boldsymbol{\mu M})$} & \multicolumn{2}{c}{ MIC $(\boldsymbol{\mu M})$} \\
\hline & A549 & MRC5 & S. aureus & P. aeruginosa PAO1 \\
\hline Perrottetin F (1) & $15^{\mathrm{a}}$ & 30 & 100 & 150 \\
$\mathbf{2}$ & 25 & 70 & 450 & 200 \\
$\mathbf{3}$ & 50 & 80 & 400 & 250 \\
$\mathbf{4}$ & 250 & 250 & 400 & 250 \\
\hline
\end{tabular}

${ }^{a}$ The results are expressed as means calculated from three independent experiments with standard deviation between $1-3 \%$.

Next, we focused on antibacterial potential of perrottetin F (1) and its three biotransformed products against two common nosocomial opportunistic pathogens: $S$. aureus and $P$. aeruginosa PAOI (Table 2). Very weak antibacterial activities against $S$. aureus and $P$. aeruginosa PAO1 with minimal inhibitory concentrations (MICs) ranging from $100 \mu \mathrm{M}$ for perrottetin $\mathrm{F}$ to $450 \mu \mathrm{M}$ for 2 were recorded. Again, the activity of biotransformants was 1.3- to 4.5-fold reduced in comparison to the parent compound (Table 2).

The effect of compounds (2-4) on bacterial pigment production was assessed using $C$. violaceum CV026 and S. marcesens (Figure S37, Supporting Information). C. violaceum CV026 is white, violacein negative, and has no constitutive ability of violacein pigment production, but CV026 can sense exogenously added AHLs and responds by producing purple violacein [18]. In this assay, the tested compounds demonstrated only weak antagonistic activity on violacein production in $C$. violaceum CV026 when $250 \mu \mathrm{g}$ of test compound was applied per disc. Inhibition of violacein production with $\mathbf{2}$ and $\mathbf{3}$ was visible as a slightly brighter area around the discs (Figure S37A, Supporting Information). The interference of perrottetin $\mathrm{F}$ and biotransformants with pigment production in $S$. marcescens was examined by assessing modulation of prodigiosin production in a disc assay (Figure S37B, Supporting Information). It was obvious from the obtained results that compounds did not trigger inhibition of prodigiosin synthesis, however, added compounds could change the color of the halo around the discs, from red to pink. The pink zones were larger in the presence of biotransformats than in the presence of parent compound perrottetin F (1) (Figure S37B, Supporting Information). Taken together, obtained results with $C$. violaceum $\mathrm{CV} 026$ and $S$. marcesens 
suggested that perrottetin $\mathrm{F}$ as well as three metabolites (2-4) had moderate effect on violacein and prodigiosin production under tested conditions.

S. marcescens has N-acylhomoserine lactones (AHLs)- dependent QS system and produces at least four AHLs (3-oxo-C6-HSL, C6-HSL, C7-HSL, and C8-HSL), which regulate production of prodigiosin and phenotypic characteristics such as motility and biofilm formation that have been connected to virulence of this strain [28]. On the other side, P. aeruginosa also possesses four QS systems: two acyl-homoserine lactone (AHL)-mediated QS systems, one 2-alkyl-4-quinolones (AHQs)-mediated QS system and the IQS-dependent QS system. Three autoinducer synthases LasI, RhlI, and PqsABCDH produce autoinducers 3-oxo-C12-homoserine lactone (HSL), C4-HSL, and 2heptyl-3-hydroxy-4-quinolone, respectively. To evaluate the potential of the perrottetin F (1) and biotransformed products 2-4 modulators of specific QS pathway in $P$. aeruginosa, three mutant biosensors strains dependent on exogenous AHLs were used: $P$. aeruginosa PA14-R3 $\Delta$ lasIPrsaI::lux, used to determine 3OC12-HSL production (LasI activity), P. aeruginosa PAO1 $\Delta$ rhlIpKD-rhlA, used for C4-HSL production (RhlI activity), and $P$. aeruginosa PAO1 $\Delta$ pqsA mini-CTX luxPpqsA, used for assessment of PQS production (PqsABCDH activity).

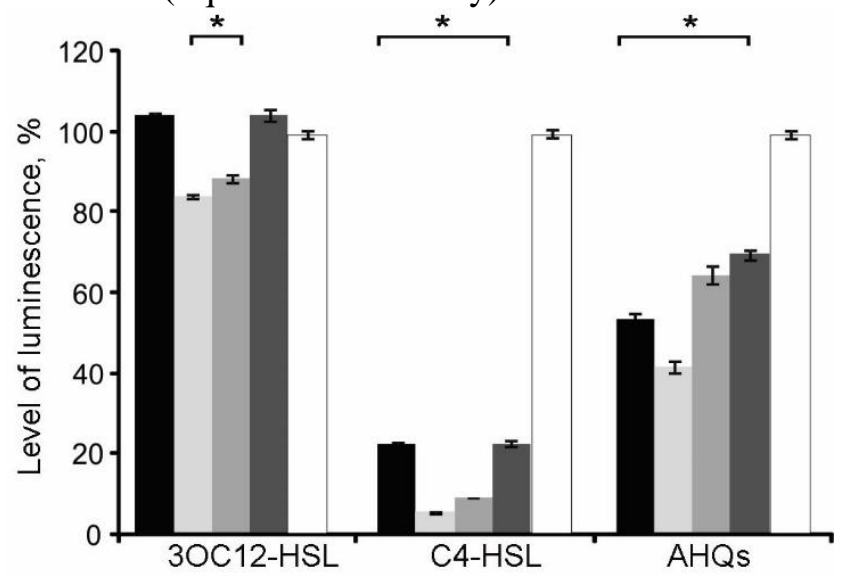

Figure 2. Effect of perrottetin F and compounds 2-4 $(100 \mu \mathrm{M})$ on acyl homoserine lactone (AHL) and AHQs production in $P$. aeruginosa. The $P$. aeruginosa PA14- R3 $\Delta$ lasIPrsal : lux strain was used to directly measure the levels of 3OC12- HSL and P. aeruginosa PAO1 $\triangle$ rhlIpKD-rhlA reporter strain was used to directly measure the levels of C4-HSL. Detection of AHQs (PQS and HHQ) was performed by the biosensor $P$. aeruginosa PAO1 $\triangle$ pqsA mini-CTX luxPpqsA. Biosensors grown in the presence of DMSO served as the $100 \%$ luminescence control ( $\square$ ); $\mathbf{\square}$ - perrottetin $\mathrm{F}(\mathbf{1}), \square-\mathbf{2}, \square-\mathbf{3}$, $\square-4$

Obtained data (Figure 2) suggest that perrottetin $\mathrm{F}$ and biotransformants reduced emitted bioluminescence in biosensor strains, in a range of $10 \%$ to $90 \%$ compared to negative control (DMSO). All these compounds exerted the most potent effects on C4-HSL (Rhl activity), moderate effects on AHQs production (PqsABCDH activity) and weak effects on 3OC12-HSL production (Las activity). Biotransformed products, especially $\mathbf{2}$ and $\mathbf{3}$, had more pronounced effect in comparison to parent perrottetin F. Compounds $\mathbf{2}$ and $\mathbf{3}$ significantly reduced the bioluminescence in $P$. aeruginosa PAO1 $\triangle$ rhlIpKD-rhlA negatively for $95 \%$ and $90 \%$, respectively, in comparison to DMSO control (Figure 2). Also, these compounds significantly reduced the bioluminescence in $P$. aeruginosa PAO1 $\triangle$ pqsAmini-CTX luxPpqsA for $60 \%$ and 35\%, respectively, and in addition, reduced bioluminescence in $P$. aeruginosa PA14-R3 $\Delta$ lasIPrsal::lux, for $16 \%$ and $12 \%$ respectively. Perrottetin F (1) and compound 4 inhibited the bioluminescence in $P$. aeruginosa PAO1 $\triangle$ rhlIpKD-rhlA for $80 \%$, and negatively affected the bioluminescence in $P$. aeruginosa PAO1 $\triangle$ pqsAmini-CTX luxPpqsA for 50\% and $30 \%$, respectively, while these compounds had no effect on the bioluminescence in $P$. aeruginosa PA14-R3 $\Delta$ lasIPrsaI::lux.

Taken together, these results suggested that the tested compounds, that showed no significant effect on the growth of $P$. aeruginosa and $S$. aureus (Table 2), were able to successfully modulate the production of signaling molecules that are part of the bacterial QS system (Figure 2).

According to literature data, acyclic bis-bibenzl, perrottetin $F(\mathbf{1})$ and cyclic bis-bibenzyls, marchantins $\mathrm{A}, \mathrm{B}$, and $\mathrm{D}$ showed cytotoxicity against $\mathrm{KB}$ cells $(3.7-20 \mu \mathrm{M})$, DNA polymerase $\beta$ 
inhibitory ( $\left.\mathrm{ID}_{50} \quad 14.4-97.5 \mu \mathrm{M}\right)$, and anti-HIV-1 $(5.30-23.7 \mu \mathrm{g} / \mathrm{mL})$ activity [29]. Perrotetin F exhibited very strong nitric oxide (NO) production inhibitory activity, in culture media, on RWA 264.7 cells [4]. Perrottetin F also inhibited influenza PA (subunit of RNA polymerase) endonuclease activity at $10 \mu \mathrm{M}[4,8]$. Perrottetin E, bis-bibenzyl isolated from Radula perrottetii inhibited KB cells at a concentration of $12.5 \mu \mathrm{g} / \mathrm{mL}$ [4].

A series of bis-bibenzyls, including riccardin-, marchantin- and isoplagiochin-class structures were evaluated for anti-MRSA activity against two strains of $S$. aureus MRSA [30]. The structureactivity relationships and the results of molecular dynamics simulations showed that bis-bibenzyls with potent anti-MRSA activity commonly have a 4-hydroxyl group at the D-benzene ring and a 2hydroxyl group at the C-benzene ring in the hydrophilic part of the molecule, and an unsubstituted phenoxyphenyl group in the hydrophobic part of the molecule containing the A-B-benzene rings. It was found previously that the number of hydroxyl groups on the riccardin framework is important for anti-MRSA activity, thus, bis-bibenzyls derivative with hydroxyl group did not show apparent antiMRSA activity, whereas bis-bibenzyls with two or three phenolic hydroxyl groups showed antiMRSA activity. Also, it was shown that riccardin-class bis(bibenzyl)-type cyclic phenolic compounds exhibit potent antibacterial activity towards MRSA S. aureus, comparable to that of vancomycin and linezolid [30]. Cyclic bis-bibenzyl, marchantin A from many Marchantia species, showed antibacterial activity against Bacillus cereus (MIC $12.5 \mu \mathrm{g} / \mathrm{mL})$, B. megaterium $(25 \mu \mathrm{g} / \mathrm{mL})$, B. subtilis $(25 \mu \mathrm{g} / \mathrm{mL})$, Cryptococcus neoformans $(12.5 \mu \mathrm{g} / \mathrm{mL})$, Escherichia coli, Pseudomonas aeruginosa and Staphylococcus aureus $(3.13-25 \mu \mathrm{g} / \mathrm{mL})[4]$.

AHL-mediated systems regulate the expression of many virulence factors, including LasA protease, phospholipase, exotoxin A, pyocyanin, rhamnolipids, elastase, and also regulate biofilms development. Pseudomonas aeruginosa PAO1 are known to produces a diverse 2-alkyl-4-quinolones (AHQs) which act as QS signal molecules and are involved in the regulation of many virulence factors. In the quinolone-dependent QS system, PqsABCDE and PqsH are involved in the synthesis of 2-heptyl-4-quinolone (HHQ) and 2-heptyl-3-hydroxy-4-quinolone (PQS) [23]. Both HHQ and PQS can bind to the transcriptional regulator MvfR and form a complex to regulate gene expression. $P$. aeruginosa $\mathrm{PQS}$ and HHQ regulate the production of pyocyanin, rhamnolipids, elastase and influence a biofilm formation [28]. The production of diverse AHQ, which act as QS signal molecules and are also involved in the regulation of many virulence factors in $P$. aeruginosa PAO1 (i.e. elastase, pyocyanin, rhamnolipids, biofilm development).

Biotransformation of bis-bibenzyls has not yet been investigated. Biotransformed products of these molecules found in the most thalloid liverworts could be useful in the study of antimicrobial, anticancer and other biologically active compounds. The microbial transformation is safe, cheap and constitutes an environmentally very friendly method which could be a starting platform for the synthesis of new pharmacological drugs. In the present research, three new bis-bibenzyl derivatives are obtained through biotransformation of perrottetin $\mathrm{F}$ by $A$. niger and their cytotoxic and antimicrobial properties were tested. Moreover, biotransformants showed an extraordinary ability to inhibit synthesis of bacterial quorum-sensing signal molecules such as short chain acyl homoserine lactones (AHLs).

\section{Acknowledgments}

This work was financially supported by the Ministry of Education, Science and Technological Development of Republic of Serbia (Grant Nos. 451-03-68/2020- 14/200178, 451-03-68/202014/200026 and 451-03-68/2020-14/200042). The authors thank The Matsumae International Foundation for the financial support and post doc position at Tokushima Bunri University, Japan for D.B).

\section{Supporting Information}

Supporting information accompanies this paper on http://www.acgpubs.org/journal/records-of$\underline{\text { natural-products }}$ 


\section{ORCID}

Danka Bukvicki 0000-0001-6807-0038

Miroslav Novakovic 0000-0002-4984-041X

Tatjana Ilic-Tomic 0000-0001-7198-2855

Jasmina Nikodinovic-Runic 0000-0002-2553-977X

Nina Todorovic 0000-0002-7798-9449

Milan Veljic 0000-0002-1779-1721

Yoshinori Asakawa 0000-0002-1847-412X

\section{References}

[1] Y. Asakawa (1982). Chemical constituents of the Hepaticae. Springer: Progress in the Chemistry of Organic Natural Products, Vienna.

[2] Y. Asakawa, M. Toyota, R. Matsuda, K. Takikawa and T. Takemoto (1983). Distribution of novel cyclic bisbibenzyls in Marchantia and Riccardia species, Phytochemistry 22, 1413-1415.

[3] Y. Asakawa (1993). Biologically active terpenoids and aromatic compounds from liverworts and the inedible mushroom Cryptoporus volvatus. In: Bioactive Natural Products: Detection, isolation, and structural determination. eds: Colegate SM, Molyneux RJ, CRC Press, Boca Raton, Florida, pp. 319-347.

[4] Y. Askawa (2013). Ludwiczuk A, Nagashima F. In Progress in the Chemistry of Organic Natural Products; eds: Kinghorn, D. A., Falk. H., Kobayashi. J. Springer: Vienna, 95, 1-796

[5] Y. Asakawa (2016). Polyphenols in bryophytes: structures, biological activities, and bio- and total syntheses. In Recent Advances in Polyphenol Research, eds: Yoshida K, Cheynier V, Quideau S, WileyBlackwell, UK, pp. 36-66.

[6] M. Toyota, M. Tori, K. Takikawa, Y. Shiobara, M. Kodama and Y. Asakawa (1985). Perrottetins E, F, and $\mathrm{G}$ from (liverwort)-isolation, structure determination, and synthesis of perrottetin E, Tetrahedron Lett. 26, 6097-6100.

[7] M. Novakovic, D. Bukvicki, B. Andjelkovic, T. Ilic-Tomic, M. Veljic, V. Tesevic and Y. Asakawa (2019). Cytotoxic activity of riccardin and perrottetin derivatives from the liverwort Lunularia cruciata, J. Nat. Prod. 82, 694-701.

[8] Y. Asakawa, A. Ludwiczuk, F. Nagashima, M. Toyota, T. Hashimoto, M. Tori, Y. Fukuyama and L. Harinantenaina (2009). Bryophytes: Bio- and chemical diversity, bioactivity and chemosystematics, Heterocycles 77, 99-150.

[9] N. Ghani, H. N. Ismail, Y. Noma and Y. Asakawa (2017). Microbial transformation of some natural and synthetic aromatic compounds by fungi: Aspergillus and Neurospora strains. Nat. Prod. Commun. 12, 1237-1240.

[10] Y. Iwai, K. Murakami, Y. Gomi, T. Hashimoto, Y. Asakawa, Y. Okuno, T. Ishikawa, D. Hatakeyama, N. Echigo and T. Kuzuhara (2011). Anti-Influenza activity of marchantins, macrocyclic bisbibenzyls contained in liverworts, PLoS One. 6(5):e19825

[11] C. F. Xie, H. Q. Yuan, J. B. Qu, J. Xing, B. B. Lü, X. N. Wang, M. Ji and H. X. Lou. (2009). Biocatalytic production of acyclic bis[bibenzyls] from dihydroresveratrol by crude Momordica charantia peroxidase, Chem. Biodivers. 6, 1193-1201.

[12] W. He, J. Wang, L. Zhang and Z. Liu (2012). Biotransformation of ginsenosides and their aglycones, Int. J. Biomed. Pharm. Sci. 6(1), 45-55.

[13] R. Azerad (1999). Microbial models for drug metabolism. In: Biotransformations, Advances in Biochemical Engineering/Biotechnology, ed: Faber K. Springer, Berlin, Heidelberg, pp.169-218.

[14] B. Mutafova and S. Mutafov (2016). Microbial transformations of plant origin compounds as a step in preparation of highly valuable pharmaceuticals, J. Drug Metab. Toxicol. 7, 1-11.

[15] G. Chen, M. Yang, S. Nong, X. Yang, Y. Ling, D. Wang, X. Wang and W. Zhang (2013). Microbial transformation of 20(S)-protopanaxadiol by Absidia corymbifera. Cytotoxic activity of the metabolites against human prostate cancer cells, Fitoterapia 84, 6-10.

[16] Y. Asakawa and A. Ludwiczuk (2018). Chemical constituents of bryophytes: Structures and biological activity, J. Nat. Prod, 81, 641-660.

[17] M. B. Hansen, S. E. Nielsen and K. Berg (1989). Re-examination and further development of a precise and rapid dye method for measuring cell growth/cell kill. J. Immunol. Methods. 119, 203-210.

[18] K. H McClean, M. K. Winson, L. Fish, A. Taylor, S. R. Chhabra, M. Camara, M. Daykin, J. H. Lamb, S. Swift, B. W. Bycroft, G. S. A. B. Stewart and P. Williams (1997). Quorum sensing and Chromobacterium violaceum: exploitation of violacein production and inhibition for the detection of $\mathrm{N}$-acylhomoserine lactones, Microbiology 14, 3703-3711. 
[19] J. H. Merritt, D. E. Kadouri and G. A. O’Toole (2005). Growing and analyzing static biofilms. In: Current Protocols in Microbiology. John Wiley \& Sons, Inc., Hoboken, NJ, USA.

[20] T. Ilic-Tomic, M. Sokovic, S. Vojnovic, A. Ciric, M. Veljic, J. Nikodinovic-Runic and M. Novakovic (2016). Diarylheptanoids from Alnus viridis ssp. viridis and Alnus glutinosa: Modulation of quorum sensing activity in Pseudomonas aeruginosa, Planta Med. 83, 117-125.

[21] K. Duan and G. Surette (2007). Environmental Regulation of Pseudomonas aeruginosa PAO1 Las and Rhl quorum-sensing systems, J. Bacteriol. 189, 4827-4836.

[22] F. Massai, F. Imperi, S. Quattrucci, E. Zennaro, P. Visca and L. Leoni (2011). A multitask biosensor for micro-volumetric detection of N-3-oxo-dodecanoyl-homoserine lactone quorum sensing signal, Biosens Bioelectron. 26, 3444-3449.

[23] M. P. Fletcher, S.P. Diggle, S. A. Crusz, S.R. Chhabra, M. Cámara and P. Williams (2007). A dual biosensor for 2-alkyl-4-quinolone quorum-sensing signal molecules, Environ. Microbiol. 9, 2683-2693.

[24] K.H.C. Baser and G. Buchbauer Handbook of essential oils, science, technology, and applications, CRC Press 2009, Print ISBN: 978-1- 4200-6315-8, eBook ISBN: 978-1-4200-6316-5.

[25] M. Novakovic, D. Bukvicki, V. Vajs, T. Tesevic, S. Milosavljevic, P. Marin and Y. Asakawa (2018). Microbial transformation of Calamintha glandulosa essential oil by Aspergillus niger, Nat. Prod. Commun. 13, 479-482.

[26] G. Jard, T. Liboz, F. Mathieu, A. Guyonvarch, F. Andre, M. Delaforge and A. Lebrihi (2010). Transformation of zearalenone to zearalenone-sulfate by Aspergillus spp, World Mycotoxin J. 3, $183-191$.

[27] J. Y. Kwak, L. K. Seok, H. Suh, Y.H. Choi, S. S. Hong, D. S. Kim and Y. C. (2016). Boo Antimelanogenic effects of luteolin 7-sulfate isolated from Phyllospadix iwatensis Makino. Br J. Dermatol. 175, 501-511

[28] J. Lee and L. Zhang (2015). The hierarchy quorum sensing network in Pseudomonas aeruginosa, Protein Cell. 6, 26-41.

[29] Y. Asakawa (2007). Biologically active compounds from bryophytes. Pure Appl. Chem. 79, 557-580.

[30] H. Sawada, K. Onoda, D. Morita, E Ishitsubo, K. Matsuno, H. Tokiwa, T. Kuroda and H. Miyachi (2013). Structure-anti MRSA activity relationship of macrocyclic bis(bibenzyl) derivatives, Bioorg. Med. Chem. Lett. 23, 6563-6568.

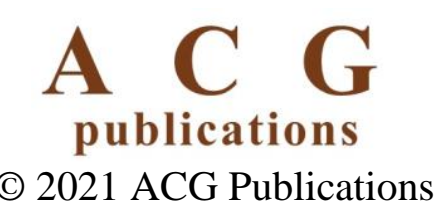

